\title{
Technical Term Extraction Using Measures of Neology
}

\author{
Christopher Norman \\ Royal Institute of Technology \\ The University of Tokyo \\ chnorakth.se
}

\author{
Akiko Aizawa \\ National Institute of Informatics \\ The University of Tokyo \\ aizawa@ni.ac.jp
}

\begin{abstract}
This study aims to show that frequency of occurrence over time for technical terms and keyphrases differs from general language terms in the sense that technical terms and keyphrases show a strong tendency to be recent coinage, and that this difference can be exploited for the automatic identification and extraction of technical terms and keyphrases. To this end, we propose two features extracted from temporally labelled datasets designed to capture surface level $n$-gram neology. Our analysis shows that these features, calculated over consecutive bigrams, are highly indicative of technical terms and keyphrases, which suggests that both technical terms and keyphrases are strongly biased to be surface level neologisms. Finally, we evaluate the proposed features on a gold-standard dataset for technical term extraction and show that the proposed features are comparable or superior to a number of features commonly used for technical term extraction.
\end{abstract}

\section{Introduction}

Keyphrases are terms assigned to documents, conventionally by its authors, that are intended chiefly as an aid in searching large collections of documents, as well as to give a brief overview of the document's contents. Technical terms are words or phrases that hold a specific meaning in specific domains or communities. Keyphrases are closely related to technical terms in the sense that the keyphrases assigned to a document are generally selected from the terminology of the document's domain. Keyphrases and technical terms show considerable conceptual overlap, and by extension, so do keyphrase and technical term extraction. As a consequence, these two are closely related research topics. In this study we will see technical term extraction and keyphrase extraction as distinct but related. We will take the view that the technical terms in a scientific article are likely candidates to be keyphrases for the document and consequently that technical term extraction methods might also be useful in keyphrase extraction.

We will show that features that capture the neology of term candidates can be used to extract technical terms, and that the basic assumptions that enable this extraction also hold true for keyphrases.

This paper is organized as follows: We first discuss how technical terms and keyphrases differ from general language terms in terms of neology. We then define features that capture this difference and analyze these features statistically using the SemEval-2010 dataset (Kim et al., 2010) and a gold-standard for technical term extraction derived from the same dataset (Chaimongkol and Aizawa, 2013). Our analysis shows that the proposed features reliably separate positive from negative examples, both of technical terms and of keyphrases. Furthermore, the histograms for the proposed features are very similar when calculated for technical terms and keyphrases, suggesting that technical terms and keyphrases have very similar neological properties. Finally, we demonstrate that this statistical bias can be used to reliably extract technical terms in a gold-standard dataset, and that the proposed features are comparable or superior to other features used in technical term extraction, with an F-score of 0.509 as compared to 0.593 , $0.367,0.361$, and 0.204 for affix patterns, tf-idf, word shape, and POS tags respectively.

We argue that, given the high performance of the proposed features on technical term extraction, and given that we can show that the statistical properties that enable us to use them to extract technical terms also extend to keyphrases, the proposed features should also be useful in keyphrase extraction. 


\section{Related works}

Most technical term extraction systems work fairly similarly to keyphrase extraction systems, using an initial $n$-gram or POS tag-based filtering to identify term candidates, then proceeding to narrow this list down using machine learning algorithms on various kinds of document statistics such as term frequency or the DICE coefficient (Justeson and Katz, 1995; Frantzi et al., 2000; Pinnis et al., 2012). For an in-depth summary of the state-of-the-art in technical term extraction, we refer to Vivaldi and Rodríguez (2007). For a summary of the state-of-the-art in keyphrase extraction, which largely follow the same pattern, we refer to Hasan and $\mathrm{Ng}$ (2014). The main difference in implementation might simply come down to a choice in top-level machine learning approach: in technical term extraction it makes sense to view the problem as a binary classification problem, whereas in keyphrase extraction it makes more sense to see the problem as a ranking problem.

Approaches based on frequency statistics extracted from the documents themselves are, however, not without their drawbacks. To begin with, for document statistics to be meaningful we will need a dataset that is large enough, consisting of documents that are large enough individually. We might also encounter problems if the documents are too large, because then the statistics might be drowned out by noise in the data (Hasan and $\mathrm{Ng}$, 2014). We should also be careful about the topical composition of the data set - if the dataset only contains documents from a single domain, then we will have to approach the problem very differently than if the dataset contains documents from multiple domains. Preferably, we want methods that do not make these kinds of assumptions about the data set, methods that can be applied to documents of any size, and to document collections of any size or of any topical composition. In the best of worlds, we want methods that can be applied to document collections consisting of a single document, or even a single sentence.

One way to go beyond simple document statistics is to use external, pregenerated resources. To give some examples of this, Medelyan and Witten (2006) use a pregenerated domain thesaurus to conflate equivalent terms and to select candidates that are thematically related to each other, Hulth et al. (2006) use a pregenerated domain ontology to select candidates whose synonyms, hypernyms, and hyponyms also appear in the text, and Lopez and Romary (2010) use a terminology database as one way to measure the salience of term candidates for keyphrase extraction in scientific articles. Medelyan et al. (2009) use a somewhat more indirect external resource by taking the frequency by which a term candidate appears in Wikipedia links, divided by the frequency by which it appears in Wikipedia documents. The idea behind using external resources is that human annotators generally perform better than automatic systems, and resources produced by human beings are thus much more reliable than automatic methods, even if the resources themselves are only obliquely related to keyphrases.

However, depending on the speed by which the terminology of the subject field changes, any previously generated resource might become outdated very quickly. In a subject field such as law, where the terminology only changes imperceptibly over time (Lemmens, 2011), this is unlikely to be an issue, but in a quickly changing subject field such as information science, where the terminology has been reported to change by as much as $4 \%$ per year (Harris, 1979), it is likely that pregenerated resources will lag behind recent terminological developments. One selling point of the automatic extraction of keyphrases or technical terms is that automatic methods are able to respond to changes in the terminology of a subject field with the same speed that the terminology changes, but if we rely on pregenerated resources then we forsake this advantage, since these are unlikely to include terminology that only recently appeared in the subject field.

\section{Theoretical basis}

In this paper we will examine the use of external corpora in order to track the frequency of occurrence of $n$-grams over time, and use measures of neology as a way to extract technical terms. We are not aware of any previous attempts to use neology as a feature for technical term extraction, keyphrase extraction, or other kinds of natural language processing tasks. We will use the Google Ngrams dataset (Lin et al., 2012), where this information is already extracted. Although we use this dataset, mainly because of its convenience in our initial investigation, there is nothing keeping us from using other corpora consisting of raw documents, such as Pubmed. In particular, this 
would allow us to obtain more recent data than the Google Ngrams dataset, which only contains frequencies of occurrence from before 2008.

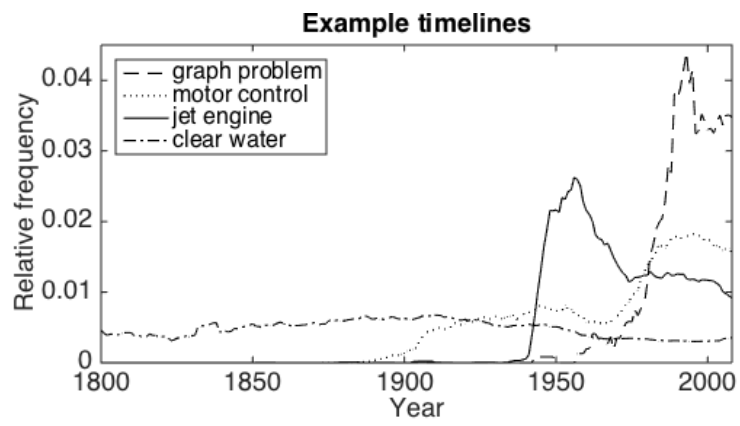

Figure 1: Example timelines (frequency of occurrence) for three technical terms and one nontechnical term. The frequencies of occurrence for each timeline has been normalized to sum to one to fit all graphs in the same diagram.

In order to develop some intuition about how technical terms are adopted, let us look at the timelines for some technical terms in the Google Ngrams dataset (Figure 1). To begin with, all the technical terms (graph problem, motor control, and jet engine) are relatively recent coinage, and none of them were in use in the 19th century. In all three cases, there is some point in time at which the term gained momentum and began to surge in frequency. This characteristic is fairly typical of technical terms, although we can of course find general language terms that exhibit the same pattern of adoption. By contrast, the non-technical term (clear water) has been in use throughout the 19th and 20th century. Unlike technical terms, general language do not have generally observable characteristics, and the shape of the timelines vary greatly from case to case. The defining characteristic instead seems to be one of contrast: general language terms seldom have the steep curves that we can observe of the technical terms here.

We will formalize this difference and examine it statistically in the later parts of this study.

Of course, our ability to find neologisms by examining the frequency of occurrence of their surface forms necessitates that technical terms generally do not share surface forms with general language terms. If such is the case, then the general language senses of the terms are likely to drown out the technical term senses. For instance, consider a term like worm in computer security. The vast majority of the occurrences of the unigram worm in the Google Ngrams dataset are likely to be of the biological variety, and it is consequently impossible to tell from the Google Ngrams dataset alone that the computer security term only appeared in the later half of the 20 th century ${ }^{1}$. Fortunately, the case where technical terms coincide with general language terms is rare, at least when considering terms composed of multiple words.

The overall recency of coinage of technical terms depends on the subject field - the majority of the terminology in e.g. computer science consists of terms whose surface forms were introduced no earlier than the middle of the 20th century, whereas subject fields such as mathematics and physics include terminology coined in the 19th century or earlier. Consequently, if we plot the frequency of usage of a neologism over time we would expect to see a curve similar to those in Figure 1, but we should expect that the curves may be shifted to the left or to the right, largely depending on the subject field.

Why are technical terms so often neologisms? It turns out that general language, which is what is ordinarily studied in linguistics, and special language, which is what we actually encounter in the documents commonly used for keyphrase extraction, differ quite substantially in linguistic aspects (Sager et al., 1980). One difference is that surface level neologisms are seldom created in general language. Rather, the creation of new surface forms generally occur in special language, from which the term might later be transferred to general language (Sager et al., 1980, p. 287). Consequently, terms that have appeared recently, given some specific point in time, are likely to be domain-specific at that point. The longer that has passed since the adoption of the term, the more likely it is that the term has been adopted into general language.

\section{Measures of neology}

We have noted that the shape of the timelines seem to indicate whether a given term is recent coinage, but in order to use these as input to machine learning algorithms, we need to distill the high dimensional data into low-dimensional features that retain the neological information.

\footnotetext{
${ }^{1}$ However, the term computer worm is a surface level neology. We might thus observe that new senses of the unigram worm appeared in the later half of the 19th century by examining bigrams.
} 
What we want to extract is of course not necessarily the shape of the timelines, but whether the occurrences of the $n$-grams predominantly occur in the far right side on the time axis. In other words, we want to determine if the timeline is mainly concentrated on the right side. This is simple to do using statistical measures such as the mean and the standard deviation of the curves.

Let $f_{y}^{i}$ denote the frequency of an $n$-gram $i$ in year $y$. Then $p_{i}(y)=\frac{f_{y}^{i}}{\Sigma_{y} f_{y}^{i}}$ constitutes a probability density function, with the expected value:

$$
\mu_{i}=\sum_{y} p_{i}(y) \cdot y=\frac{\sum_{y} f_{y}^{i} \cdot y}{\sum_{y} f_{y}^{i}}
$$

How this "mean" should be interpreted might not be completely intuitively obvious, but for our purposes here it is enough to note that $\mu_{i}$ indicates where the curve is mainly concentrated. If the curve is concentrated around higher values of $y$ then we have, by definition, a surface level neologism.

We can take the standard deviation of $p_{i}$ in the same way:

$$
\sigma_{i}^{2}=\sum_{y} p_{i}(y) \cdot\left(y-\mu_{i}\right)^{2}=\frac{\sum_{y} f_{y}^{i} \cdot\left(y-\mu_{i}\right)^{2}}{\sum_{y} f_{y}^{i}}
$$

The standard deviation $\sigma_{i}$ then yields a measure of how much the probability density is concentrated around $\mu_{i}$, in other words, how "steep" the probability density is. A low standard deviation consequently indicates that the term has been adopted or abandoned rapidly. Low standard deviation should thus in general imply either surface level neologisms or fads. If we are only interested in how quickly a term has been adopted and not how quickly it might have been abandoned, then we can take a one-sided "standard deviation", by separating out the $y$ for which $y<\mu_{i}$, but this does not seem to make much difference for the sake of the separability of technical terms. Those terms that have been adopted quickly also appear to be likely to quickly fall into relative disuse.

We should hasten to point out that there does not seem to exist any theoretical reasons to use the mean and standard deviation in this way. For instance, using the peak of the curve (i.e. the mode of the distribution) might have more intuitive appeal, since this should correspond to the point in time at which the term was in its most widespread use. However, the Google Ngrams dataset is of- ten plagued by severe noise, in particular for less commonly used $n$-grams such as technical terms, and the peaks of the timelines are thus likely to be spurious. The mean and the standard deviation may be crude measures of shape, but they have the advantage of being robust against noise, and can generally be used with good results even for very noisy timelines.

Other intuitively appealing features, such as the first order derivatives of the timelines or the skewness of $p_{i}$ have turned out not to be very useful, presumably because of the noise.

\section{Statistical properties of technical terms and keyphrases}

In this section, we examine statistically the features we propose, and show that both technical terms and keyphrases are strongly biased towards certain values for our features. We also underline the relationship between technical terms and keyphrases by showing that these are very similar in terms of neology.

To analyze keyphrases we will use the SemEval-2010 dataset (Kim et al., 2010), one of the most commonly used gold standards for keyphrase extraction. To analyze technical terms we will use a dataset consisting of the abstracts from the SemEval-2010 dataset manually annotated by two annotators such that all the technical term spans have been labeled (Chaimongkol and Aizawa, 2013). Since this dataset was constructed from the abstracts of the SemEval-2010 dataset we assume that these datasets are similar enough that analyzing and comparing the statistical properties of these two datasets is meaningful.

For the analysis in the following section, we construct three classes of data:

- We extract the $n$-grams that are part of the spans labeled as technical terms in the Chaimongkol-Aizawa dataset to obtain one set of positive examples of technical terms.

- We extract the constituent $n$-grams from the gold-standard keyphrases from the SemEval2010 training dataset (using the combined set) to obtain one set of positive examples of keyphrases

- We extract the $n$-grams that are not part of the spans labeled as technical terms in the Chaimongkol-Aizawa dataset to obtain one 

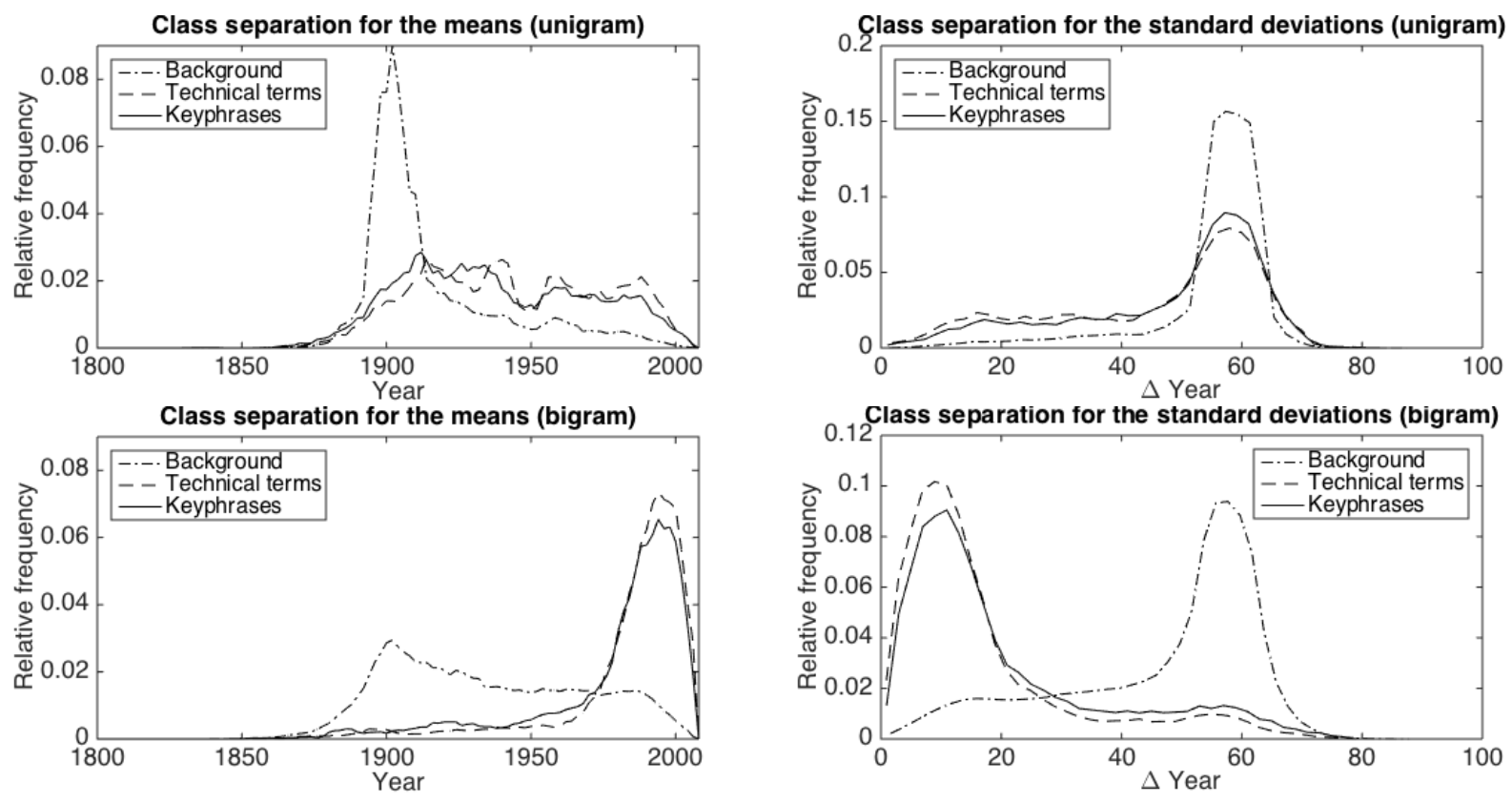

Figure 2: Class separation between technical terms, keyphrases, and background terms over $\mu_{i}$ (left) and $\sigma_{i}$ (right), when considering unigrams (top), and bigrams (bottom). Histogram bin size was set to 2 in all cases. The resulting histograms have been smoothed using Matlab's default settings (moving average with span 5) and normalized to sum to one.

set of negative examples of both technical terms and keyphrases.

We could of course extract negative examples of keyphrases from the SemEval-2010 dataset, but it is not so clear that these would all unquestionably be negative examples of keyphrases. Given that inter-annotator agreement is generally very low for keyphrases, this set might well contain terms that could reasonably be considered keyphrases by other annotators. We here assume that the negative examples of technical terms also constitute negative examples of keyphrases, and that this set is less likely to contain borderline cases of keyphrases. Only using three classes of data also simplifies both the exposition and the processing.

For these three classes of $n$-grams, we extract the corresponding timelines from the Google Ngrams dataset over the period 1800-2008, and we calculate the means $\mu$ and standard deviations $\sigma$ from these as defined in the preceding section. To analyze the features $\mu$ and $\sigma$ we plot the histograms of the values for the technical term $n$ grams and the keyphrase $n$-grams versus the background $n$-grams (Figure 2).

In order for the features to be useful for either technical term extraction or keyphrase extraction we would like to see as little overlap as possible between the histograms of the positive and the negative examples. This seems to hold true for the bigrams, but not for the unigrams. In the unigram case we can see only a weak tendential difference in the histogram densities of the positive and negative examples.

We should point out that the mode of the histogram densities for the negative examples fall very close to the mean and standard deviation of a uniform distribution over the period 1800-2008. These would occur around 1904 and 60.48 respectively.

In all cases, the histograms for the technical terms and the keyphrases are very similar. This might not seem very surprising given that all the data is derived from the SemEval-2010 dataset, but it bears mentioning that these were annotated by different people, and more importantly, using very different annotation criteria.

We omit trigrams and higher order $n$-grams from consideration.

It is unlikely that higher order $n$-grams would help in keyphrase and technical term extraction, because the Google Ngrams dataset excludes any $n$-gram that has a total frequency of occurrence less than 50. This means that less frequently used 
$n$-grams, such as technical terms, as well as higher order $n$-grams are likely to be missing (see Table 1). This problem is not very severe for unigrams and bigrams, but technical term trigrams suffer from data sparsity problem severe enough to essentially render them useless. Part of this problem might be due to the large mismatch between the dataset used for evaluation, and the Google Ngram dataset used to identify neology. If we were to use an external dataset from a more similar domain to the evaluation set, then we would expect to find a greater portion of the $n$-grams in the external dataset.

\begin{tabular}{rcc} 
& Technical terms & Background \\
\hline Unigram & $97.5 \%$ & $100 \%$ \\
Bigram & $85.0 \%$ & $97.0 \%$ \\
Trigram & $25.5 \%$ & $71.0 \%$
\end{tabular}

Table 1: The ratio of $n$-gram in each class in the Chaimongkol-Aizawa dataset that occur in the Google Ngrams dataset. The percentages have been generated by chosing a random sample of 200 unigrams of each class, 200 bigrams of each etc., and checking if the $n$-gram occur in the Google Ngrams dataset using the web interface.

\section{Evaluation on technical term extraction}

In order to demonstrate that neology, as characterized by the features $\mu$ and $\sigma$, can be used to automatically extract technical terms, we implement a simple technical term extractor using these as features. We generally follow the approach taken by Chaimongkol and Aizawa (2013) and implement a conditional random field model to BIO-tag the dataset. The major difference in implementation is that we use the neology features extracted from Google Ngrams in the term extractor, and that we do not use features based on clustering.

For the sake of our CRF model, bigrams and unigrams are sufficient, since what we want to do is to obtain features corresponding to each node (i.e. to each unigram) and features corresponding to the links between the nodes (i.e. to each bigram). We might in theory achieve better performance with higher order $n$-grams, but in reality the results would be severely hampered by the sparsity problems for higher order $n$-grams.

Similarly to Chaimongkol and Aizawa, we implement a CRF model using the freely available state-of-the-art CRF framework CRFSuite ${ }^{2}$, using five different feature sets:

1. POS TAGS using the Stanford POS tagger ${ }^{3}$.

2. WORD SHAPE features extracted similarly to Chaimongkol and Aizawa. These include binary features such as whether the current token is capitalized, uppercased, or alphanumeric.

3. AfFIXES of length up to 4 characters extracted for all tokens. In other words, for the token carbonization we would extract carb-, car-, ca-, $c_{-}$, -tion, -ion, -on, and - $n$.

4. TF-IDF for each unigram and bigram in the dataset.

5. NeOLOGY based features, in other words the mean and standard deviation of the Google Ngrams timeline as described in section 4.

The mutual information between each neighboring token in the dataset has also been tried, but this turned out to not have any perceptible effect on the results.

Because CRFSuite cannot handle continuous features, such as tf-idf, $\mu$, or $\sigma$, we had to resort to discretizing these by binning. Appropriate bin sizes were established experimentally.

We apply the system on the labeled dataset where we attempt to binary classify each token into positive and negative examples, where positive examples are those that are part of a technical term compound, and negative those that are part of the background. We use the full dataset, and evaluate using 10-fold cross-validation.

Using all features, the system achieves an Fscore around 0.7 for the technical term tokens, and an F-score around 0.9 for the non-technical term tokens.

To compare the different features with each other, we evaluate their performance individually (Table 2). The best performing feature turns out to be the affixes, although our neology features are quite comparable in performance. Neology performs better than all other features except affixes.

\footnotetext{
${ }^{2}$ http: //www. chokkan.org/software/ crfsuite/

${ }^{3}$ http://nlp.stanford.edu/software/ tagger.shtml
} 


\begin{tabular}{rcccccc} 
& \multicolumn{3}{c}{ Technical terms } & \multicolumn{3}{c}{ Non-technical terms } \\
& $\mathrm{P}$ & $\mathrm{R}$ & $\mathrm{F}_{1}$ & $\mathrm{P}$ & $\mathrm{R}$ & $\mathrm{F}_{1}$ \\
\hline POS tags & $\mathbf{0 . 7 3 4}$ & 0.118 & 0.204 & 0.835 & $\mathbf{0 . 9 9 1}$ & 0.906 \\
Word shape & 0.659 & 0.248 & 0.361 & 0.853 & 0.971 & 0.909 \\
Affixes & 0.673 & $\mathbf{0 . 5 3 0}$ & $\mathbf{0 . 5 9 3}$ & $\mathbf{0 . 9 0 0}$ & 0.943 & $\mathbf{0 . 9 2 1}$ \\
tf-idf & 0.600 & 0.244 & 0.367 & 0.852 & 0.964 & 0.904 \\
Neology & 0.637 & 0.423 & 0.509 & 0.881 & 0.947 & 0.913
\end{tabular}

Table 2: Term extractor performance in terms of correctly labeled tokens. Here, the system is only using a single feature class in each trial in order to compare the relative performance of each feature class.

\begin{tabular}{rcccccc} 
& \multicolumn{3}{c}{ Technical terms } & \multicolumn{3}{c}{ Non-technical terms } \\
& $\mathrm{P}$ & $\mathrm{R}$ & $\mathrm{F}_{1}$ & $\mathrm{P}$ & $\mathrm{R}$ & $\mathrm{F}_{1}$ \\
\hline All features & 0.728 & 0.673 & 0.700 & 0.929 & 0.944 & 0.936 \\
- POS tags & 0.728 & 0.656 & 0.690 & 0.925 & 0.946 & 0.935 \\
- Word shape & 0.719 & 0.671 & 0.694 & 0.928 & 0.942 & 0.935 \\
- Affixes & $\mathbf{0 . 6 9 1}$ & $\mathbf{0 . 6 3 4}$ & $\mathbf{0 . 6 6 1}$ & $\mathbf{0 . 9 2 0}$ & $\mathbf{0 . 9 3 7}$ & $\mathbf{0 . 9 2 9}$ \\
- tf-idf & 0.717 & 0.643 & 0.678 & 0.923 & 0.944 & 0.933 \\
- Neology & 0.715 & 0.647 & 0.679 & 0.923 & 0.943 & 0.933
\end{tabular}

Table 3: Term extractor performance in terms of correctly labeled tokens. Here, the system is using all but one feature class in each trial in order to compare the relative performance drop when each feature class is removed from the classifier.

It might be mentioned that these results are calculated over all tokens, even those where the neology features are missing because the corresponding $n$-grams do not occur in the Google Ngrams dataset. It is likely that the performance of the neology features would be higher if these were excluded from consideration. This might seem like cheating, but we should consider what would happen if we were to use another dataset with greater coverage, or some future improved version of the Google Ngrams dataset with greater coverage.

We also perform an ablation experiment to see how much the performance drops when excluding individual feature classes (Table 3). Similarly, the biggest drop occurs when excluding affixes. In this case, however, the differences between the different features are quite modest, which seems to imply that each single feature does not contain much information that is not also contained in the other features.

Compared to tf-idf, the neology feature is often able to correctly identify technical term spans containing terms which are also frequent in the remainder of the document collection, such as technical terms containing words like: network, computer, function, algorithm, complexity, data, server, model, or vector. It is much less easy to summarize where neology works well compared to the other features besides tf-idf.

Neology features are much less effective when the technical terms coincide with general language terms, for instance worm, precision, or MAP. This is generally only a problem in the unigram case, and bigrams such as computer worm or average precision generally do not have this problem.

\section{Discussion}

In this paper we have shown that technical terms tend to be recently coined, and that this statistical tendency is strong enough that it allows us to extract technical terms with reasonable accuracy. We have also shown that this statistical feature of technical terms also seem to hold true for keyphrases, and we therefore maintain that it is reasonable that similar features might also be useful in keyphrase extraction. We should not expect equally high performance in keyphrase extraction, however, since in keyphrase extraction we are not only interested in whether the output keyphrases are terms in the relevant domain, but also that they are significant in the document under consideration. What we do suggest is that neology can be useful for keyphrase extraction when used in concert with other features such as tf-idf that indicate significance or topicality.

The extraction of either technical terms or 
keyphrases fundamentally depends upon the assumption that these are biased in certain ways. For instance, a common assumption taken in keyphrase extraction is that keyphrases are biased to occur more frequently at certain positions in the document. Another common assumption is that technical terms and keyphrases are biased to occur with different frequencies in certain communities, or that the contexts in which keyphrases and technical terms appear differ between different communities. Similarly to the position and community bias of keyphrases, we suggest that keyphrases also have a time bias, that the keyphrases of a document are skewed to be overrepresented in the contemporary and subsequent literature, but likely to be absent or severely underrepresented in the precedent literature.

The very high values of the means, and the very low values of the standard deviations observed for the technical terms in section 5 suggests that the majority of the technical term bigrams studied in this paper come from terms that only appeared after 1950 . This might be explained by the fact that the datasets we use here are derived from the SemEval-2010 dataset, which is strongly biased towards computer science literature. It seems reasonable that the separation between the classes should generally be stronger in subject fields where the terminology tends to be very recent coinage than in fields with more mature terminology. If this is true, then the approach we propose here should work well for subject fields where the terminology is rapidly changing, and where the need for automatic extraction methods is arguably the greatest.

\section{Acknowledgements}

This work was supported by the Grant-in-Aid for Scientific Research (B) (15H02754) of the Japan Society for the Promotion of Science (JSPS).

\section{References}

Panot Chaimongkol and Akiko Aizawa. 2013. Utilizing LDA Clustering for Technical Term Extraction. Proceedings of the Nineteenth Annual Meeting of the Association for Natural Language Processing, Nagoya, pages 686-689.

Katerina Frantzi, Sophia Ananiadou, and Hideki Mima. 2000. Automatic recognition of multi-word terms: The C-value/NC-value method. International Journal on Digital Libraries, 3:115-130.
Jessica Harris. 1979. Terminology change: Effect on index vocabularies. Information Processing \& Management, 15(2):77-88.

Kazi S. Hasan and Vincent Ng. 2014. Automatic Keyphrase Extraction : A Survey of the State of the Art. Acl, pages 1262-1273.

Anette Hulth, Jussi Karlgren, and Anna Jonsson. 2006. Automatic keyword extraction using domain knowledge. Computational Linguistics and Intelligent Text Processing, pages 472-482.

John S. Justeson and Slava M. Katz. 1995. Technical terminology: some linguistic properties and an algorithm for identification in text. Natural Language Engineering, 1:9-27.

Su Nam Kim, Olena Medelyan, Min-Yen Kan, and Timothy Baldwin. 2010. Semeval-2010 task 5: Automatic keyphrase extraction from scientific articles. Proceedings of the 5th International Workshop on Semantic Evaluation, pages 21-26.

Koen Lemmens. 2011. The slow dynamics of legal language: Festina lente? Terminology, 17:74-93.

Yuri Lin, Jean-baptiste Michel, Erez L. Aiden, Jon Orwant, Will Brockman, and Slav Petrov. 2012. Syntactic Annotations for the Google Books Ngram Corpus. Proc. of the Annual Meeting of the Association for Computational Linguistics, pages 169-174.

Patrice Lopez and Laurent Romary. 2010. HUMB : Automatic Key Term Extraction from Scientific Articles in GROBID. Proceedings of the 5th International Workshop on Semantic Evaluation, pages 248-251.

Olena. Medelyan and Ian H. Witten. 2006. Thesaurus based automatic keyphrase indexing. Proceedings of the 6th ACM/IEEE-CS joint conference on Digital libraries, pages 6-7.

Olena Medelyan, Eibe Frank, and Ian H. Witten. 2009. Human-competitive tagging using automatic keyphrase extraction. Proceedings of the 2009 Conference on Empirical Methods in Natural Language Processing, 3:1318-1327.

Mārcis Pinnis, Nikola Ljubešić, Dan Ştefănescu, Inguna Skadiņa, Marko Tadić, and Tatiana Gornostay. 2012. Term Extraction, Tagging, and Mapping Tools for Under-Resourced Languages. Proceedings of the 10th Conference on Terminology and Knowledge Engineering, pages 193-208.

Juan C. Sager, David Dungworth, and Peter F. McDonald. 1980. English special languages: principles and practice in science and technology. John Benjamins Publishing Company.

Jorge Vivaldi and Horacio Rodríguez. 2007. Evaluation of terms and term extraction systems: a practical approach. Terminology, 13:225-248. 